\title{
Prototype Design of Social Networking APP Based on Context of Tourism
}

\author{
Zhuan Wei, Chang-li Qu \\ Digital Publishing and Printing college, Changsha Normal University, Xinsha, Changsha, Hunan, China
}

\begin{abstract}
The study aims to desgin social networking mobile application for tourism users with mobile devices by their context. By practical research methods, such as online questionnaires, telephone interviews and user interviews, user habits and needs were digged out. The paper simulated and reproduced the process of user traveling by scenario analysis method, then extracted the context. Final prototype design of social networking application on context of tourism is approved that it can meet the demands of the user personalization and improve user satisfaction.
\end{abstract}

Keywords - context, travel, social networking, APP(application)

\section{基于情境的旅游社交网络 APP 原型设计}

\author{
魏专 権昌利
}

长沙师范学院数字出版印刷系, 星沙, 长沙, 湖南, 中国

摘 要 研究针对旅游用户移动设备使用情境设计的社交网络手机应用软件方法。通过网上调查问卷、电话采访和用户访谈等实际 调研的方法, 挖掘用户习惯和需求。使用剧情分析的方法来模拟再现用户旅行过程, 提取情境。运用情境感知理论设计的手机旅游社 交 APP 及其用户界面。最终设计出的旅游社交网络 APP 原型能满足用户个性化需求并提升用户满意度。

关键词 情境, 旅游, 社交网络, APP

\section{1. 引言}

随着互联网和通信技术, 特别是移动互联网的发展, 手机移动社交网络应用如 WeChat、QQ 等已经融入到我 们生活的各个方面。情境感知通过系统感应器对用户目 前情境进行感知, 并做出相应的反应, 极大的满足了用 户的使用体验需求。然而计算机领域的情景感知是通过 感知计算和系统的自适应来实现的, 能否运用设计方法 来完成情景感知后的用户界面设计是本研究要解决的问 题。

\section{2. 研究背景}

情境感知的移动手机界面能自动的感知用户的使用 模式和行为习惯并自动调整界面, 其中感知的过程主要 是通过智能手机内部的各种传感器来获取用户生理特 征、手势、甚至是意图信息, 然后通过动态的改变界面
以方便用户操作和提升用户交互体验。用户界面设计研 究涉及到心理学、艺术设计、视觉设计等相关知识。国 外在用户界面设计领域方面主要集中在用户体验设计方 面, 2003 年 LoriK.Shir[1]做了关于界面设计的可用性测 试及其在用户满意度和用户偏好的效果的研究, 2004 年 Anthony Savidis[2]等人研究了普及的交互样式来统一用 户界面设计，以形成多样化的界面样式，2012 年 Chelsea.Chase[3]也提出用户界面设计要使用设计样式, 强调在界面设计中利用设计样式能设计出更加人性化 的、流畅的界面, 而在移动网络领域中, 布莱恩[4]研究 出基于移动平台的界面设计技术; Josh Clark[5]通过发掘 iphone 用户习惯特征总结出设计的方法。国内对界面设 计的研究随着移动网络发展而发展, 相对比国外要晚。 2011 年王俊[6]提出一套用于移动应用交互设计的方法, 对 APP 界面设计有很好的借鉴意义。与此同时基于情境

湖南省教育厅项目支持（项目号：13C1066）；湖南省大学生研究项目支持（项目号：2014/604）；湖南省科技厅项目支持（项目号： 2013TZ2022) 
感知的界面设计也有了相应的研究, 何秀琴[7]在 2012 年 研究了基于情境感知的手机自适应用户界面设计, 创新 的提出了一种新的情境感知自适应界面的模型, 程时伟 [8]等人也对情景感知自适应用户界面进行了相关的研 究, 认为自适应界面能提高用户的操作效率和改善用户 体验感受。杨焕[9]在 2013 年总结提炼出一套专门针对智 能手机的界面设计方法。纵观国内外 APP 界面设计研究 尚处于初步阶段, 基本集中在交互设计和视觉设计的概 念性原理中 [10]。本研究期望运用情境感知理论设计一款 手机旅游社交 APP, 用设计方法进行用户体验研究并改 进用户界面, 满足用户个性化需求, 提升用户满意度。

\section{3. 产品定位}

随着自助游的流行和智能手机的普及, 具有旅游协 助和移动社交功能的手机 APP 越来越受到旅游爱好者的 欢迎, 开发设计的 APP 产品希望能利用智能手机的照相 机、麦克风、定位感应器等情境感知设备定位分析游客 在旅途中的轨迹以及记录游客在不同旅游点的体验感 受, 并根据情境感知记录为游客推送其可能喜好的附近 景点, 除此之外, APP 还具备移动社交网络功能, 可以 在旅途中和旅行结束后分享旅行图片和旅途感受, 并进 行网络互动, 此款 APP 命名为“游多多”, 寓意对旅游协 助功能多多。

开发设计 APP 产品需要考虑不同的运行环境和操作 系统, 目前最主要的手机操作系统有三个: Android、iOS、 和 Windows phone。Android 是 Google 开发的基于 Linux 平台的开源手机操作系统, 也是迄今为止应用最为广泛 的手机操作系统, 其现在已经建立成了一个标准化、开 放式的移动电话软件生态系统; iOS 是由苹果公司开发的 移动操作系统, 现在主要在 iPhone 上使用, iOS 与苹果 的 MacOSX 操作系统一样, 属于类 Unix 的商业操作系统; Windows Phone (简称: WP) 是微软发布的一款手机操 作系统, 目前也有在一些手机中使用。本设计面向 iOS 系统的进行开发, 完成的 APP 可以在 iPod touch、iPad 以及 Apple TV 等产品上运行。

\section{4. 情境分析}

\section{1 用户需求分析}

用户需求以界面设计为目的, 通过了解用户特殊使 用情境挖掘用户潜在需求。研究利用网上调查问卷、电 话采访和用户访谈等实际调研的方法, 获取 APP 功能、 用户人群、用户界面、用户习惯等用户情境数据, 再利 用统计学的方法进行数据分析, 得出用户在不同情境下 的特征、习惯和需求。需求分析得出的结论是用户需要
一款用于旅行游记、拍摄美照, 并上传到网络社区进行 分享、约游出行、聊天交谈、分享心情等的社交应用软 件, 从而满足了用户和志同道合的人一起旅游及互动的 需求。对用户需求分析可以整理出 APP 的几个主要功能 模块: 聊天、分享、约游、导航、推荐旅游景点、和附 近的人玩游戏等。

\section{2 用户情境分析}

旅游过程中涉及到的情景信息很多, 不同环境下用 户情景相互交织相当复杂, 其中涉及到的情景包括: 用 户人数、旅游时间、旅游地址、用户地理位置、用户喜 好、旅游景点气温、景点地理位置、景点门票价格、景 点介绍等, 如果按照情境类型来进行划分, 游客在旅途 中的情境可以分为三大类: 用户情境、景点情境和环境 情境。具体采用剧情分析的方法来模拟再现整个旅行过 程, 从中挖掘整理相关情境。

剧情 1: 小王, 男, 20 岁, 湖南工业大学在读大学 生, 五一假期准备去云南丽江旅游, 手机预装“游多 多”APP, 出发前一个星期在“游多多”分享社区查看了网 友的游记, 于是开始在注册用户中寻找有共同意愿有共 同时间去丽江的株洲网友, 最终约好三人周六早上出发, 11 点多到达丽江, 利用 APP 查找附件特色客栈, 并办理 入住手续。下午 2 点, 几人在丽江街头游玩, 打开“游多 多”APP 使用拍照功能, 以景点为背景进行自拍, 完成后 APP 通过情境感知和情境计算, 将照片上传共享, 并自 动跳转到景点的详情介绍页面, 包括了景点的历史、特 色、介绍等。晚上 7 点在一酒吧坐下来喝一杯, 听流浪 歌手们唱歌儿, 可以通过 APP 点歌, 结束后入住客栈, 通过 APP 自动识别所定房间。

剧情 2: 小李, 女, 31 岁, 外地人来广州工作, 平 时工作忙没时间, 准备利用清明节一天的假期去广州市 内游玩, 不愿查攻略不愿跟团, 想走到哪看到哪。早上 8 点驾车出发去南越王墓博物馆, 利用“游多多”APP 语音 导航功能, 界面自动切换到导航地图界面, 并通过后台 情境计算为他分配了一条最近的路线。看完南越王墓博 物馆接近中午, 天气炎热, 打开 APP 查找附近的好评饭 店和小吃。吃完饭后用 APP 查看附件景点, 后台一直在 记录小李的路径和周围的地理信息、环境信息, 通过计 算分析他去过的景点类别, 为他推荐适合的景点。接着 去了中山纪念堂, 每人联票 25 元。最后利用 APP 景点推 荐和导航到最近的景点陈家祠, 在进景区之前询问了一 下景点的历史, 景点的票价是 10 元。最后, 在 APP 指引 下驾车回家。

通过剧情分析可以对游客使用 APP 的情境有详细的 
了解。剧情 1 和剧情 2 都有手机拍照拍照的情境, 两个 剧情中都通过手机自身的情境感知设备进行识别, 剧情 1 情境感知方式有位置感知、用户动作感知等; 在剧情 2 情境感知方式有地理位置的感知、语音感知、用户喜好 感知等。手机设备中能够感知用户使用情境的方式有很 多, 比如常见的录音设备、视频设备、重力感应系统、 GPS 系统等等, 这些方式可以感知到用户的地理位置、 语音、人脸、使用动作等个性特征, 从而掌握用户当时 的使用情境。在此基础上, 用户界面、系统交互设计根 据情境做出适应性调整, 从而更好的调整系统功能结构 和进行任务指引。

\section{3 系统功能设计}

游多多是一款应用于旅游并和驴友互动交流的社交 类 APP, 除了在旅游过程中使用之外, 还可以用于旅游 出行前的约游和路线规划。系统功能设计利用专业软件 MindManager 构建, MindManager 是由美国 Mindjet 公司 开发的一款创造、管理和交流思想的通用标准的绘图软 件, 可以进行 APP 总体设计、界面规划、模块划分、系 统接口设计、逻辑流程设计等。根据用户在旅游中的情 境分析, APP 由四个主要功能模块组成: 探索、分享、 发现、个人。此外还有一个快捷模块, 以便快速调出常 用的工具, 如相机和地图。默认开启的是探索界面, 探 索界面有两大模块: 附近和目的地, 其中不同景点可以 相互切换, 附近可以进行附近活动、语音搜索、地图定 位、好友分布情况等相关搜索, 目的地包括搜索框、目 的地推荐和留言; 发现界面包括附近游戏、闪拍、游趣 分享、拼游、附近小组等功能模块; 分享界面包含预览、 图片墙、旅程编辑、积攒等功能模块; 个人界面主要是 对个人信息进行设定并进行地理位置定位等。

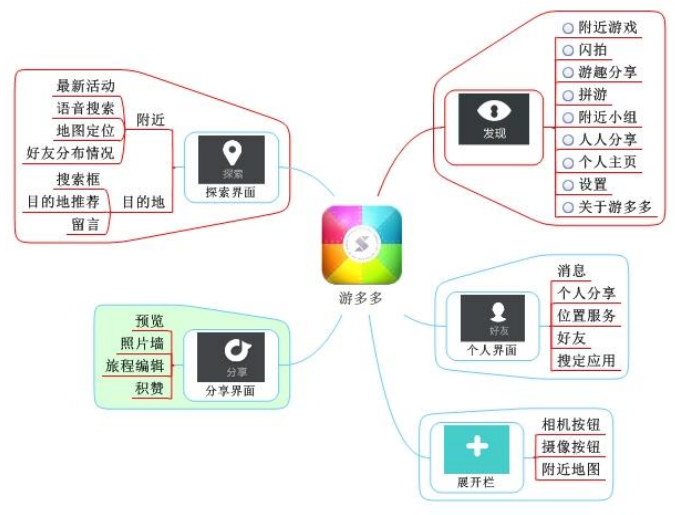

图 1 “游多多”APP 功能架构图

\section{5. 系统原型设计}

完整的 APP 系统设计流程包括低保真原型设计、高 保真原型设计、技术开发和系统执行。因为本设计研究 是从设计角度进行的思考, 技术开发和系统执行交由协 同合作的系统工程师完成, 这里不再累述。

\section{1 低保真原型设计}

低保真原型设计是对产品较简单的模拟, 它基本停 留在产品的外部特征和功能构架上, 可以通过简单的设 计工具迅速制作出来, 用于表现最初的设计概念和思路。 因此, 它通常被用于设计过程初期的需求收集和分析, 简单的产品原型可以作为设计开发人员与用户的沟通载 体, 帮助用户表达其对产品的期望和要求, 但通常不能 实现与用户的互动。低保真原型使用到的 SKETCH 软件 快速绘制。

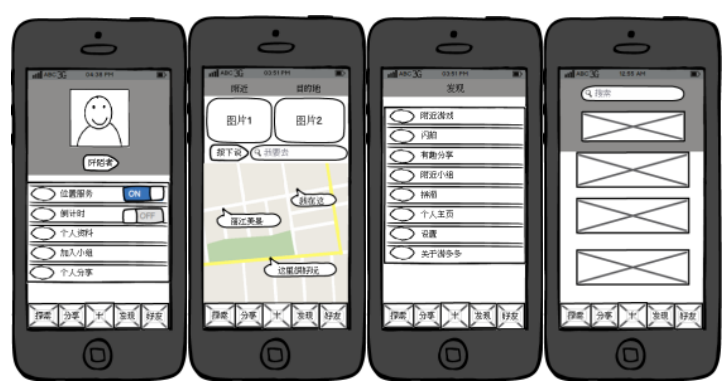

$\mathrm{a}$ 个人界面 $\mathrm{b}$ 探索界面 $\mathrm{c}$ 发现界面 $\mathrm{d}$ 分享界面

图 2 “游多多”低保真原型图

\section{2 高保真原型设计}

高保真原型设计是高功能性、高互动性的原型设计, 它可以忠实显示产品和界面主要或全部的功能和工作流 程, 具有完全的互动性, 使用户可以像使用真实产品一 样完成各种任务, 例如数据的输入和输出、菜单选择、 导航汶览等等。高保真产品原型的开发通常要消耗大量 的时间和精力, 它往往被用于需求分析之后的细节设计 和使用性评估来发现产品在互动性和工作流程方面的使 用性问题。高保真原型设计软件使用 Axure。

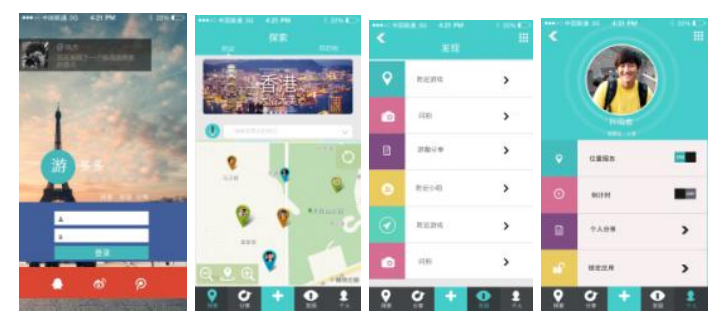

$\mathrm{a}$ 个人界面 $\mathrm{b}$ 探索界面 $\mathrm{c}$ 发现界面 $\mathrm{d}$ 分享界面

图 3 “游多多”高保真原型图 


\section{6. 结语}

情境感知对于移动设备的应用设计十分重要, 利用 设计的方法对感知到的旅游过程中的用户情境进行分析 并融合到移动设备应用的原型设计和界面设计中，可以 很好的为用户进行个性化设计, 并做出适应性的调整, 对于产品开发阶段意义重大。随着情境和情境感知的进

一步运用, 未来的移动设备应用设计将更加的智能化和 更具友好性。

\section{参考文献(References)}

[1] Lori K. Shird. Examining Usability and its Effects on User Satisfaction and User Preferences for Interface Design. Nacogdoches: Austin State University, 2003:35-42.

[2] Anthony Savidis,Constantine Stephanidis.Unified User Interface Design: Designing Universally Accessible Interactionsp. Computers in Human Behavior, 2004,16(3): 243-270.

[3] Chelsea Chase. Using Design Patterns in User Interface Design. Cincinnati: University of Cincinnati, 2012:1-36.
[4] Brian. Design and development of mobile applications. Beijing: electronic industry press, 2010:11-16, 23.

[5] Josh Clark. Tapworthy: Designing Great iPhone Apps. Sebastopol: O'Reilly Media, 2011:56-75.

[6] Wang junwork. Facing the availability of the smart phone software interaction design research. $\mathrm{Xi}$ 'an: northwest university, 2011:1-69.

[7] He Xiuqin. Situational awareness based mobile adaptive user interface design research. Beijing: Beijing university of posts and telecommunications, 2012:11-13.

[8] Cheng wei, xiao-jian liu, shou-qian sun. Situational awareness drive mobile device adaptive user interface model. Chinese journal of image and graphics, 2010, (7) : 56-59.

[9] Yang Huan. Intelligent mobile Internet application interface design research. Wuhan: wuhan university of technology, 2013:61-189.

[10] Long Ying ice. Mainly guided by the user experience of APP interface design. Beijing: the northern industrial university, 2013:1-39. 